\title{
Neuer Zugang zur Quantengravitation E10: Eine fundamentale Symmetrie der Physik?
}

\author{
Hermann Nicolai | Axel Kleinschmidt
}

\begin{abstract}
Gibt es ein Symmetrieprinzip, das das Zusammenführen aller vier Fundamentalkräfte bewirken und erklären kann? Wie würde ein derartiges Prinzip unser Verständnis von Raum, Zeit und Quantenphysik verändern? Auf diese alten Fragen gibt es bis heute keine umfassende Antwort. In den letzten Jahren wurde ein neuer Zugang entwickelt, der auf einer einzigartigen mathematischen Struktur mit dem Namen 110 basiert. Er könnte einen Rahmen zur Präzisierung dieser Fragen bieten.
\end{abstract}

$\mathrm{D}$ er Erfolg der modernen Physik spiegelt sich eindrucksvoll in der Existenz von zwei Standardmodellen wider, die jeweils die Vorgänge bei den kleinsten und bei den größten Längenskalen beschreiben. Damit deckt die physikalische Beschreibung der Natur einen Bereich von subatomaren Abständen bis zum Durchmesser des sichtbaren Universums ab. In ihren jeweiligen Gültigkeitsbereichen liefern diese Standardmodelle eine genaue Beschreibung der Messdaten, sind weitreichend anwendbar und können oft Vorhersagen für neue Experimente liefern.

Im Bereich des Mikrokosmos ist es das seit Mitte der 1960er Jahre entwickelte Standardmodell der Teilchenphysik, das seither in beeindruckenden Experimenten an Teilchenbeschleunigern auf der ganzen Welt immer weiter verfeinert und bestätigt wurde. Ein neueres Beispiel für seinen Erfolg war die Vorhersage des Topquarks im Jahr 1973 durch Makoto Kobayashi und Toshihide Maskawa (Nobelpreis für Physik 2008); die Existenz dieses schweren Quarks wurde 1995 am Fermilab (Chicago, USA) experimentell nachgewiesen.

Am anderen Ende der Größenskala steht das Standardmodell der Kosmologie, das die Entwicklung unseres Universums mithilfe der Einsteinschen Allgemeinen Relativitätstheorie (ART) über einen Zeitraum von circa 13,7 Milliarden Jahren ebenso erfolgreich beschreibt. Trotz unzähliger Experimente konnten bisher keine Abweichungen von diesen grundlegenden Theorien gefunden werden.

Selbstverständlich wäre es verfrüht, in Anbetracht dieser beachtlichen Fortschritte nun das Ende der physikalischen Forschung einzuläuten. Beide Standardmodelle lassen wesentliche Fragen offen, deren Beantwortung nach allgemeiner Auffassung eine grundlegende Revision der bestehenden physikalischen Konzepte erfordern wird. In der Kosmologie hängen diese Fragen vor allem mit dem Urknall und der Frage nach dem Ursprung von Raum, Zeit und Materie zusammen. Weitere offene Fragen betreffen das Rätsel der dunklen Materie und der dunklen Energie, die Entwicklung und das Endstadium Schwarzer Löcher sowie die

Ein Ausschnitt der unendlichdimensionalen E10-Struktur, dargestellt in einer zweidimensionalen Projektion (Grafik: T. Nutma). 
beobachtete Asymmetrie zwischen Materie und Antimaterie im Weltall $[1,2]$. Ähnlich verhält es sich mit dem Standardmodell der Teilchenphysik. Es konnte bisher keine schlüssige Erklärung für das beobachtete Spektrum von Elementarteilchen, ihre Massen und die zwischen ihnen wirkenden Fundamentalkräfte liefern.

Bei allen solchen Fragen versagen die Standardmodelle. $\mathrm{Zu}$ diesen Problemen gesellt sich noch die wichtige theoretische Forderung nach der wechselseitigen Konsistenz und Kohärenz der Modelle und Theorien. Im Zentrum steht hier die Frage nach einer Theorie der Quantengravitation, die Einsteins ART und Quantenmechanik zu einer konsistenten Theorie zusammenfassen und deren innere Widersprüche überwinden soll. Dieses Thema stellt wohl die größte Herausforderung der modernen Physik dar und hat sich bisher trotz einer enormen kollektiven Anstrengung einer zufriedenstellenden Lösung entzogen. Einen Überblick der verschiedenen Zugänge gibt [3].

Um sich solchen Fragestellungen zu nähern, bietet die Physik mehrere "Werkzeuge“ an. Der wichtigste Hinweisgeber ist das Experiment, das die Grenzen gegenwärtiger Modelle aufzeigen und die physikalische Weltsicht radikal verändern kann. Hierbei mag man an das Atomspektrum und die Stabilität des Wasserstoffatoms denken. Die Entschlüsselung des Wasserstoffatoms hat - zusammen mit der Schwarzkörperstrahlung - zur Entwicklung der modernen Quantenmechanik geführt. Wichtig ist hierbei, dass es sich um ein möglichst „reines“ Experiment handelt, so dass die physikalischen Prinzipien unverfälscht und direkt zutage treten. Neben diesen echten Experimenten bieten Gedankenexperimente sowie Symmetrie- und Einfachheitsforderungen zusätzliche Einsichten und Einschränkungen bei der Konstruktion einer neuen Theorie.

\section{Symmetrien als Leitprinzip}

Die zentrale Bedeutung von Symmetrien für den Fortschritt der Physik lässt sich durch das eingangs erwähnte Topquark verdeutlichen. Die Existenz dieses schwersten Quarks wurde einzig aufgrund der Eichsymmetrie des Standardmodells vorhergesagt (siehe „Raumzeitsymmetrien und innere Symmetrien" auf S. 136), mit der alle bis 1973 bekannten Messdaten verträglich waren. Darüber hinaus hätte die Nichtexistenz des Topquarks unweigerlich die Inkonsistenz des Modells in Form einer „Anomalie“ zur Folge gehabt.

Ähnliche Aussagen gelten für die Spezielle und Allgemeine Relativitätstheorie. Dort lassen sich aufgrund von Symmetrien ebenfalls Vorhersagen machen, mit denen die Theorie steht oder fällt. Die Äquivalenz sämtlicher Inertialsysteme und die Konstanz der Lichtgeschwindigkeit führen zu Gleichungen, die symmetrisch bezüglich Lorentz-Transformationen sind, und zum Begriff des Minkowski-Raums. Das Postulat der Kovarianz der Gleichungen der Allgemeinen Relativitätstheorie unter allgemeinen Koordinatentransformationen (die Gleichungen behalten beim Wechsel des Koordinatensystems ihre Form bei) erzwingt die Ersetzung der flachen Raumzeit durch eine gekrümmte Man-

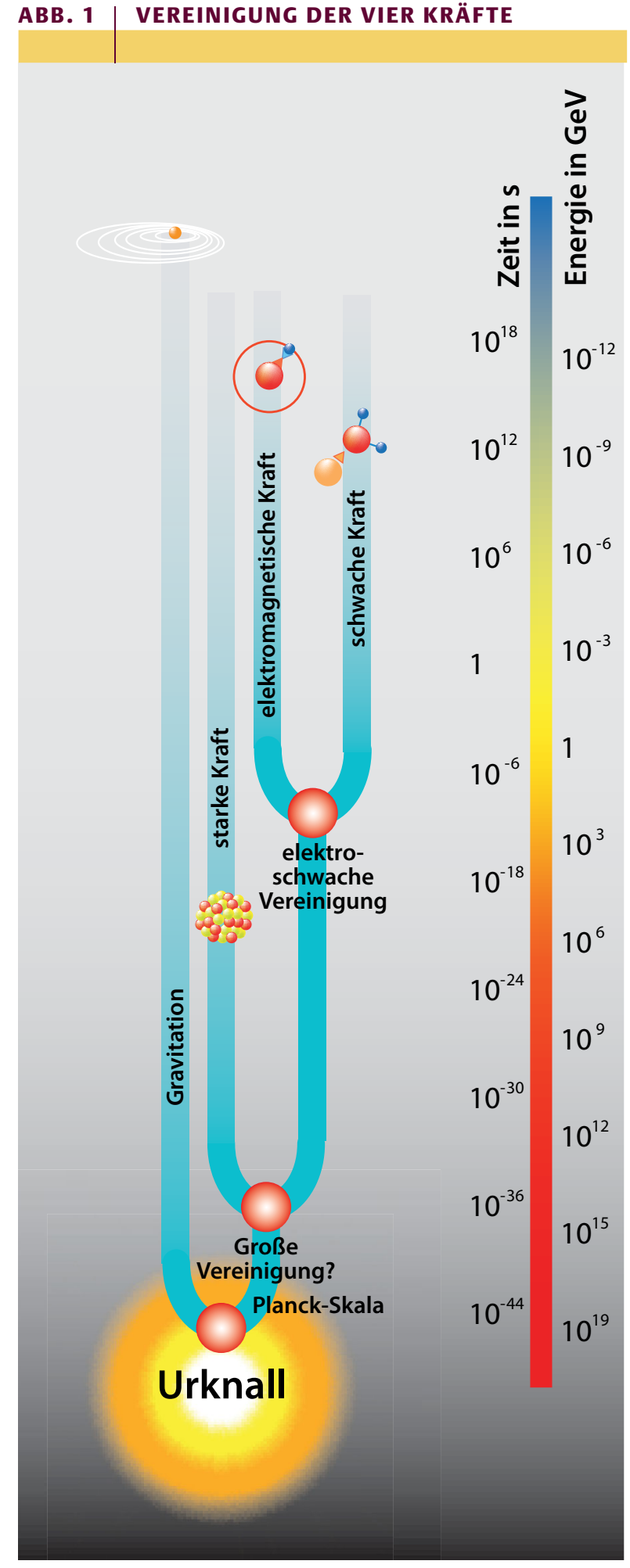

Das Diagramm zeigt die aus theoretischen Überlegungen folgende Vereinigung der vier verschiedenen Energieskalen, zusätzlich projiziert auf die Geschichte des Universums. Die Größenordnung der elektroschwachen Vereinigung ist an heutigen Teilchenbeschleunigern erreicht. Die hypothetische Vereinigung mit der starken Kraft liegt weit jenseits gegenwärtiger Technologie. Auf der Planck-Skala sollte sich die Gravitationskraft mit den anderen Kräften in einer Theorie beschreiben lassen (Grafik: R. Wengenmayr). 
nigfaltigkeit. Mit dieser erlaubt die Theorie eine rein geometrische Erklärung der Schwerkraft als Effekt der Krümmung von Raum und Zeit (siehe „Raumzeitsymmetrien und innere Symmetrien").

Es ist also eine überaus bemerkenswerte Tatsache, dass die beiden fundamentalen und erfolgreichsten Theorien der modernen Physik wesentlich durch ihre Symmetrien charakterisiert und festgelegt sind. Dem Symmetrieprinzip ordnen sich damit sämtliche beobachteten Phänomene unter.

Auch bei der Suche nach einer Theorie der Quantengravitation kommt dem Begriff der Symmetrie eine besondere Bedeutung zu: Da es momentan keine direkten Daten zu quantenmechanischen Effekten in der Gravitation gibt, fehlt das Experiment als entscheidende Richtschnur. Die relevante Längen- und Energieskala der Quantengravitation entspricht der aus heutiger Sicht wahrscheinlich fundamentalen Planck-Skala $\left(10^{-35}\right.$ m oder $\left.10^{19} \mathrm{GeV}\right)$. Sie liegt somit etwa 15 Größenordnungen jenseits der derzeit auf der Erde im Labor zugänglichen Energien [4]. So erscheint es unwahrscheinlich, dass ein Experimentum crucis existiert, mit dessen Hilfe sich die „richtige“ Theorie eindeutig identifizieren ließe - etwa in Analogie zur gewöhnlichen Quantenmechanik. Dort bestätigte erst der Vergleich der Messdaten mit den mit der Schrödinger-Gleichung berechneten

\section{RAUMZEITSYMMETRIEN UND INNERE SYMMETRIEN}

Die Physik unterscheidet grundsätzlich zwischen zwei Typen von Symmetrien. Raumzeitsymmetrien operieren in der uns umgebenden Raumzeit, zum Beispiel durch Translationen oder Rotationen im dreidimensionalen Raum. Häufig überführen solche Symmetrietransformationen Lösungen der Bewegungsgleichungen eines Systems in physikalisch andere Lösungen. Beispielsweise erzeugt ein Lorentz-Boost (Lorentz-Transformation von einem Bezugssystem in ein relativ dazu gleichförmig bewegtes ohne Rotation) eines ruhenden Teilchens ein Teilchen, das sich mit konstanter Geschwindigkeit bewegt.

Unter inneren Symmetrien versteht man dagegen Transformationen, die in einem abstrakten Hilfsraum stattfinden. Ein erstes Beispiel hierfür waren Heisenbergs Isospin-Transformationen, die Proton und Neutron ineinander „rotieren“: Wegen $m_{\text {Neutron }}$ $=1,00135 m_{\text {Proton }}$ gilt die IsospinSymmetrie in sehr guter Näherung. Ein weiteres Beispiel bilden die Eichsymmetrien, die physikalisch äquivalente Lösungen der Bewegungsgleichungen miteinander verbinden.

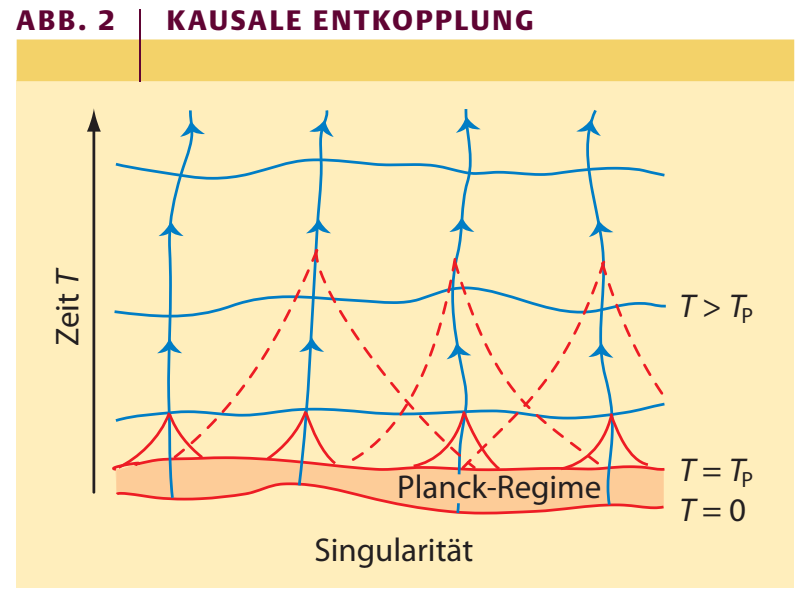

In der Nähe der Urknallsingularität zur Zeit $T=0$ findet eine kausale Entkopplung der Punkte des Raums statt (BKLLimes). Dies ist hier durch die Rückwärtslichtkegel verschiedener, beispielhaft herausgegriffener Punkte dargestellt (rote durchgezogene oder gestrichelte Linien). Ein solcher Lichtkegel enthält sämtliche Punkte, die einen gegebenen Raumzeitpunkt innerhalb des Kegels kausal beeinflusst haben können. Je näher man sich an der Singularität befindet, desto kleiner werden die kausalen Überlappungsbereiche (Übergang zu den „kleinen“ Kegeln mit durchgezogenen Linien). Dabei entkoppeln also sämtliche Raumpunkte asymptotisch. Für Zeiten unterhalb der Planck-Zeit (5 $\cdot 1^{-44}$ s) bricht die klassische Raumzeitbeschreibung zusammen (Grafikvorlage: H. Nicolai).

Spektrallinien des Wasserstoffatoms die Theorie eindeutig. Allerdings könnten indirekte Nachweise aus der Astrophysik in der Zukunft experimentelle Einschränkungen liefern, doch auch hier ist bis dato kein geeignetes Modellsystem identifiziert worden.

Anstelle eines echten Experiments kann man sich aber eines Gedankenexperiments zur Untersuchung der Struktur der Quantengravitation bedienen. Als geeignetes „Laboratorium" erweisen sich hierbei klassische Singularitäten der Raumzeit, wie sie zum Beispiel beim Urknall oder im Inneren Schwarzer Löcher auftreten. Es ist zu erwarten, dass in solchen Situationen zusätzliche Symmetrien der Gleichungen zutage treten. Dass bestimmte Grenzfälle einer physikalischen Theorie Hinweise auf Symmetrievergrößerungen liefern können, ist aus der Teilchenphysik wohlbekannt (siehe „Symmetrievergrößerung in Teilchenphysik und Kosmologie“, S. 137; Abbildung 1). Die Bedeutung von Symmetrien für die bekannten Theorien der Physik legt deshalb nahe, die Suche nach Vereinheitlichung auf das Prinzip der „Symmetrievergrößerung“ zu gründen.

Im Folgenden werden wir ausführen, wie diese Herangehensweise bei klassischen Raumzeitsingularitäten eine neuartige Symmetriestruktur impliziert. Diese Symmetrie ist unter dem Namen E10 schon seit vierzig Jahren in der Mathematik bekannt. Dort nimmt sie eine besondere Rolle in der Theorie der sogenannten hyperbolischen Kac-Moody-Algebren ein; dabei handelt es sich um eine spezielle Klasse unendlichdimensionaler Lie-Algebren. Der Begriff 
der Lie-Algebra geht auf den norwegischen Mathematiker Sophus Lie zurück. Lie-Algebren werden hauptsächlich zum Studium der kontinuierlichen Transformationen geometrischer Objekte wie zum Beispiel von differenzierbaren Mannigfaltigkeiten eingesetzt, die in der Theoretischen Physik eine wichtige Rolle spielen.

Neben der Existenz von E10 und einigen sehr allgemeinen Aussagen ist allerdings wenig über ihre Eigenschaften bekannt. Gleichzeitig hat sich jedoch herausgestellt, dass diese Struktur vieles von dem zusammenfasst, was Physiker in mehr als dreißig Jahren Superstringtheorie (meist Stringtheorie abgekürzt) [5] als möglichem Weg zu einer Quantengravitation an Wissen zusammengetragen haben. Eine E10-basierte Theorie eröffnet gleichzeitig einen neuartigen Zugang zur Quantengravitation, den wir in diesem Beitrag vorstellen wollen.

\section{Kosmologische Billards}

Stephen Hawking und Roger Penrose zeigten Anfang der 1970er Jahre, dass Raumzeitsingularitäten „generisch“ in der ART auftreten. Das bedeutet, dass eine typische Lösung der Einsteinschen Feldgleichungen einer Raumzeit entspricht, die einen Bereich unendlicher Krümmung hat. Eine besondere Klasse von Singularitäten sind sogenannte „raumartige" Singularitäten, wie zum Beispiel der Urknall. Ein wichtiges Ergebnis bei der Untersuchung solcher raumartiger Raumzeitsingularitäten geht zurück auf grundlegende Arbeiten von Wladimir Belinski, Isaak Markowitsch Chalatnikow und Jewgeni Michailowitsch Lifschitz aus den späten 1960ern und frühen 1970ern. Diese zeigten, dass sich die partiellen Differentialgleichungen der ART bei Annäherung an eine raumartige Singularität wesentlich vereinfachen.

Je näher man an einer urknallartigen Singularität ist, umso weniger haben verschiedene Punkte des Raums die Möglichkeit, miteinander kausal gekoppelt zu sein, also sich gegenseitig zu beeinflussen. Dieser sogenannte „BKL-Limes“ (nach Belinski, engl. Khalatnikov für Chalatnikow, Lifschitz) ist in Abbildung 2 anhand von Rückwärtslichtkegeln illustriert. Die kausale Entkopplung entspricht der immer geringeren Überlappung der Rückwärtslichtkegel benachbarter Punkte bei Annäherung an die Singularität. Ein verwandtes Phänomen der Kosmologie ist das sogenannte Horizontproblem, wonach naiv kausal unverbundene Bereiche des Weltalls überraschenderweise vergleichbare Eigenschaften aufweisen. Die Erklärung dieser Beobachtung im Rahmen der „inflationären“ Kosmologie ist ein Hauptmerkmal des Standardmodells der Kosmologie.

Im BKL-Limes werden auf der Ebene der Gleichungen die partiellen Differentialgleichungen der ART asymptotisch in gewöhnliche Differentialgleichungen in der Zeit überführt. Als weitere Vereinfachung verringert sich die Anzahl der dynamischen Freiheitsgrade, die in der Analyse zu berücksichtigen sind. Im Fall der standardmäßigen ART in einer vierdimensionalen Raumzeit ist nur noch die Dynamik der drei Skalenfaktoren (räumliche Ausdehnungen) des drei-

dimensionalen Raums ohne Zeitrichtung zu berücksichtigen.

Sogar die Entwicklung dieser drei Skalenfaktoren kann man noch im letzten Schritt durch ein dreidimensionales sogenanntes kosmologisches Billard annähern. Die Position der Billardkugel dieses Systems auf dem Billardtisch entspricht den räumlichen Ausdehnungen des tatsächlichen Universums. Der Billardtisch wird durch Banden begrenzt, deren genaue Lage durch die zugrunde liegenden EinsteinGleichungen mitsamt der durch sie beschriebenen Materie bestimmt ist. Für Gravitation im materiefreien Raum ist die Geometrie der Banden sehr einfach und lässt sich gut in einer zweidimensionalen Projektion auf einen hyperbolischen Raum darstellen (Abbildung 3). Die Billardkugel bewegt sich auf geraden Linien und wird von den Banden elastisch reflektiert. Oft ist die Geometrie des Tisches derart, dass ein klassisch chaotisches Verhalten resultiert (Abbildung 3).

Die Stringtheorie ist ein vielversprechender Zugang zur Quantengravitation (siehe „Stringtheorie und Supergravitation" auf S. 138). Im Grenzfall niedriger Energien lässt sie sich sehr gut durch Supergravitationstheorien beschreiben. Diese sind Verallgemeinerungen der Einsteinschen ART zu höheren Dimensionen mit festgelegtem Materiegehalt. Für die Eindeutigkeit der Materie, die an die Gravitation zu koppeln ist, sorgt die sogenannte Supersymmetrie zwischen Bosonen und Fermionen. In supersymmetrischen Theorien treten bosonische und fermionische Teilchen immer in Paaren auf. Die supersymmetrischen Partner der bekannten Teilchen sind jedoch bis dato nicht beobachtet worden -

\section{SYMMETRIEVERGRÖSSERUNG IN TEILCHENPHYSIK UND KOSMOLOGIE}

Bei den gegenwärtig zugänglichen Energien haben die drei Fundamentalkräfte der Teilchenphysik sehr unterschiedliche relative Stärken. Die sogenannten Kopplungskonstanten beschreiben diese Stärken als dimensionslose Zahlen. Die Größe der Kopplungskonstanten variiert nun jedoch mit der Energie. Mit der störungstheoretischen Berechnung dieser Abhängigkeit von der Energieskala lässt sich vorhersagen, dass bei einer Energie von etwa $10^{15} \mathrm{GeV}$ alle drei Kräfte vergleichbar stark sein sollten. Hierbei ist zu erwarten, dass die Eichsymmetrien der drei Fundamentalkräfte vollkommen verschmelzen: Je größer die Symmetrie, desto mehr Teilchen des Standardmodells treten miteinander in eine „Symmetriebeziehung“.

Dieser Prozess der Symmetrievergrößerung bei wachsender Energie (Temperatur) ist zum Beispiel die
Umkehrung des Prozesses der spontanen Magnetisierung im Ferromagneten, die sich nur bei relativ niedrigen Temperaturen ausbilden kann: Unterhalb dieser Phasenübergangstemperatur orientieren sich alle zur Magnetisierung beitragenden Elektronenspins in eine bestimmte Richtung. Folglich sind nicht mehr alle Richtungen gleichberechtigt - die Symmetrie zwischen den möglichen Spinorientierungen ist gebrochen.

Der im Standardmodell der Teilchenphysik ablaufende physikalische Prozess wird durch die Abkühlung des sich ausdehnenden Universums realisiert. Die vollständige Symmetrie einer vereinheitlichten Theorie existiert also nur bei den extrem hohen Temperaturen kurz nach dem Urknall. Symmetrievergrößerung ist auch der Grundgedanke der sogenannten Großen Vereinheitlichten Theorien (Grand Unified Theories). 
eventuell bringt der Large Hadron Collider am CERN hier neue Erkenntnisse.

Wiederholt man nun die oben beschriebene Analyse einer klassischen Raumzeitsingularität in der Supergravitation, so ergibt sich ein sehr ähnliches Bild eines kosmologischen Billards. Die maximale supersymmetrische Gravitationstheorie benötigt eine elfdimensionale Raumzeit: Entsprechend ist nun der Billardtisch nicht mehr dreidimensional, sondern zehndimensional. Wiederum lässt sich eine sehr einfache Beschreibung der Geometrie des Billardtischs finden. Er besteht aus genau zehn Banden, deren Winkel ganz bestimmte und einfache Werte annehmen.

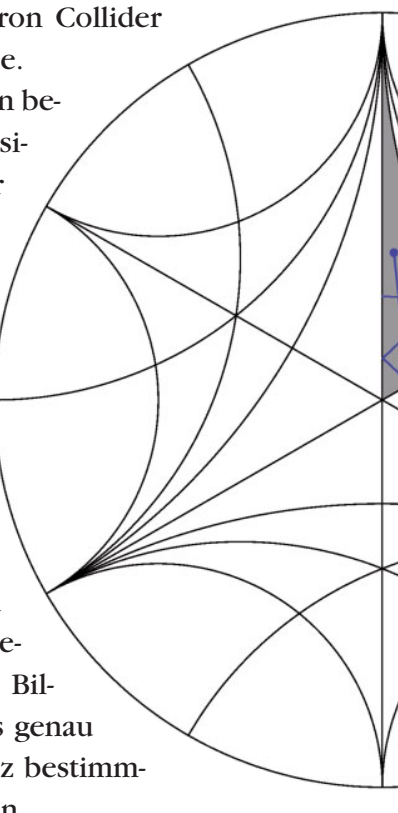

Diese Werte entsprechen nun exakt denen, die sich aus der mathematischen Struktur E10 ergeben! Das förderte die eben skizzierte Untersuchung im Jahr 2000 als große Überraschung zutage [6]. Zusammenfassend ergibt sich somit das Bild, dass in der Nähe einer Singularität der geometrische Anschauungsraum nicht mehr die richtige Beschreibung der Entwicklung liefert. Wir müssen ihn durch einen Billardtisch als Hilfsraum ersetzen, dessen Form von E10 bestimmt wird (siehe „Die E10-Struktur“ auf S. 139).

\section{E10 als fundamentale Symmetrie}

Die bei dieser Analyse gefundene Symmetrie E10 kann man folglich ernst nehmen und bei der Konstruktion einer neuen Theorie als grundlegend beibehalten. Mit dieser Annahme lässt sich ein neues Modell entwickeln, bei dem eine rein

\section{STRINGTHEORIE UND SUPERGRAVITATION}

Unter Stringtheorie versteht man im engeren Sinne Modelle, bei denen nicht elementare Punktteilchen miteinander wechselwirken, sondern eindimensionale ausgedehnte Objekte. Diese Strings kann man sich als offene oder geschlossene elastische Gummibänder vorstellen. Ähnlich einem gespanntem Gummiband oder einer Saite können Strings Schwingungen ausführen. Mit steigender Frequenz der Schwingung steigt die Energie des Strings. Durch die Äquivalenz von Masse und Energie sind höherfrequente Schwingungsanregungen massereicher.

Die unterschiedlichen Schwingungsmoden werden in der String- theorie als unterschiedliche Elementarteilchen interpretiert. Stellt man sich nun Experimente bei niedrigen Energien vor, so tragen nur wenige niederfrequente Anregungen zu Streuprozessen bei. Diese Anregungen sollten demnach den beobachteten Elementarteilchen entsprechen.

In der Tat finden sich unter den niederfrequenten Schwingungen genau solche Teilchen, die den Trägerteilchen der vier Fundamentalkräfte entsprechen. Die Dynamik dieser „leichten Stringmoden“ lässt sich bei niedrigen Energien gut durch Theorien der Supergravitation beschreiben.
Abb. 3 Kosmologisches Billard. Das dreidimensionale kosmologische Billardsystem der gewöhnlichen Allgemeinen Relativitätstheorie lässt sich auf einen hyperbolischen Raum projizieren, der unendlich viele äquivalente Billardtische enthält (Flächenstücke in der Grafik). Der grau schattierte Bereich stellt einen dieser fundamentalen Billardtische dar, auf dem die Bewegung der Kugel angedeutet ist. Sämtliche Linien sind Geodäten im hyperbolischen Raum. Das System ist chaotisch (Grafik: A. Kleinschmidt). algebraische Konstruktion an die Stelle des geometrischen Raums tritt. Dieses neue E10-Modell ist eine weniger krude Beschreibung der Singularität als im Anschauungsraum, da es mehr Freiheitsgrade als nur die räumlichen Skalenfaktoren berücksichtigt, was eine treuere Abbildung der gesamten Dynamik ermöglicht. Dies geschieht jedoch so, dass E10 in jedem Schritt als Symmetriestruktur erhalten bleibt.

In diesem Prozess wird der zehndimensionale Billardtisch nun durch einen abstrakten unendlichdimensionalen gekrümmten Raum ersetzt, auf dem sich die „Billardkugel“ nunmehr auf Geodäten (Weltlinien in höheren Dimensionen) bewegen muss. Wiederum ist zu erwarten, dass die Position der Kugel auf diesem Hilfsraum in Zusammenhang mit der Geometrie und Energiedichten der Materiebestandteile steht. Jedoch ist dieser Zusammenhang nur in speziellen Fällen exakt. Im Allgemeinen wird nämlich erwartet, dass eine geometrische Beschreibung des Universums in der Nähe einer Singularität unzulässig wird, da dort die ART nicht mehr anwendbar ist [3]. Stattdessen ist hier die algebraische E10-Sichtweise angemessen: Sie liefert eine mögliche Beschreibung der Physik im Bereich der Planck-Skala. Bevor wir uns den Konsequenzen dieser Sichtweise für die Quantengravitation zuwenden, diskutieren wir noch einige klassische Aspekte des E10-Modells [8].

Eines der wesentlichen Ergebnisse auf dem Gebiet der Superstringtheorie in den vergangenen 15 Jahren war die Entdeckung, dass die verschiedenen Stringtheorien wie über ein Netz miteinander zusammenhängen. Zuvor existierten nämlich fünf unterschiedliche Stringtheorien, und es gab keinen guten Grund, eine dieser Theorien den anderen vorzuziehen. Für die Herstellung einer einheitlichen Stringtheorie sorgte die Erkenntnis, dass es sehr indirekte $\mathrm{Zu}$ sammenhänge zwischen den fünf Theorien gibt. Diese sind unter dem Namen Dualitätssymmetrien bekannt. Als Namen für die tieferliegende Theorie hat sich der Begriff M-Theorie etabliert.

Die einfachste Manifestation einer Dualitätssymmetrie findet sich bereits in der Elektrodynamik: Obwohl bisher 
magnetische Monopole nicht nachgewiesen werden konnten, erlauben die Maxwellschen Gleichungen nach einer Vermutung von Paul Dirac aus dem Jahre 1931 zumindest im Prinzip eine neue Symmetrie, die elektrische und magnetische Ladungen untereinander vertauscht. Aus der Annahme eines magnetischen Monopols leitete Dirac auch die beobachtete Quantisierung der elektrischen Ladungen ab.

Wie sich in den letzten Jahren herausgestellt hat, können Dualitätssymmetrien darüber hinaus eine Erklärung dafür liefern, warum bei einigen Theorien das Verhalten bei schwacher dem bei starker Kopplung sehr ähnlich ist. Dualitäten machen damit Teile der Theorie der Berechnung zugänglich, bei denen die bekannten störungstheoretischen Methoden versagen - also das näherungsweise Lösen eines physikalischen Systems durch kleine Abweichungen (Störungen) von analytisch lösbaren Gleichungen.

Das E10-Modell enthält die Dualitätssymmetrien der Stringtheorien unmittelbar: Diese sind lediglich „Basiswechsel" in der Beschreibung der E10-Struktur. Somit erlaubt das E10-Modell eine direkte und systematische Realisierung dieser Idee, im Gegensatz zur konventionellen Behandlung der Stringtheorie, wo diese Symmetrien jeweils ad hoc realisiert sind. Ebenso scheint die Annahme einer E10Symmetrie viele Eigenschaften der Theorien zu implizieren, die sonst der Supersymmetrie zugeschrieben werden.

\section{E10 und Quantengravitation}

Das E10-Modell bietet einen völlig neuen Zugang zum Problem der Quantengravitation: Es ersetzt die Entwicklung der Raumzeit durch die Bewegung eines Punktteilchens auf einem unendlichdimensionalen Hilfsraum. Somit entfallen einige der üblichen Schwierigkeiten, die mit der Quantisierung der Geometrie verbunden sind. Stattdessen wird eine Quantisierung der Punktteilchendynamik angestrebt. Wegen der Komplexität der E10-Struktur wirft sie allerdings eigene, neue Fragestellungen auf.

In ersten, vereinfachten Studien untersuchte man das Problem im reduzierten Fall des zehndimensionalen kosmologischen Billards [9]. Man hoffte, dass es Hinweise auf die Eigenschaften von Quantengravitation in der Nähe einer Singularität liefern könnte. Tatsächlich fand sich, dass die Wellenfunktion des Quantenbillardsystems immer zerläuft, wenn man sich der Singularität nähert. Dies kann als ein möglicher Mechanismus zur Auflösung der Singularität aufgefasst werden.

In anderen Zugängen zur Quantengravitation, wie der Stringtheorie und der sogenannten kanonischen Quantisierung, stellen Zwangsbedingungen an die Wellenfunktion eine wesentliche Schwierigkeit dar. Im E10-Bild kann man diese Zwangsbedingungen dagegen als Einschränkung der erlaubten geodätischen Bewegungen der Billardkugel auffassen. Eine wichtige Frage ist in diesem Zusammenhang, ob die Zwangsbedingungen mit der E10-Struktur verträglich sind und eine natürliche Interpretation zulassen.

Ein wesentlich neuer Aspekt der E10-Struktur im Vergleich zu anderen Ansätzen zur Vereinheitlichung liegt mög-

licherweise darin, dass sie ein Element der Nichtberechenbarkeit (in einem mathematisch wohldefinierten Sinn) in die Quantengravitation einbringen könnte. Das wäre dann der Fall, wenn sich herausstellen sollte, dass ein vollständiges quantentheoretisches Verständnis der Urknallsingularität prinzipiell an der fehlenden vollständigen Darstellbarkeit der sie beschreibenden Gleichungen scheitern könnte.

Die E10-Struktur selbst ist durch einfache Bedingungen (Generatoren und Relationen) definiert, die jedoch im Keim ein exponentielles Wachstum an Komplexität nach sich ziehen. Auf dem Wege der Analogie mag man hierbei zum Beispiel an die berühmte Mandelbrot-Menge denken: Diese ist zwar durch einfache Gleichungen gegeben, lässt sich aber immer nur näherungsweise berechnen. Vielleicht entzieht sich der Prozess des „Verschwindens" von Raum und Zeit bei der Annäherung an die Singularität ebenso einer vollständigen mathematischen Beschreibung.

\section{Ausblick}

Gegenwärtige Untersuchungen befassen sich vorrangig mit Fragestellungen, die unmittelbar an die bisher dargestellten Ergebnisse anknüpfen.

An erster Stelle steht die dringliche Frage, inwieweit die E10-Symmetrie abseits des Urknalls erhalten bleibt, und wie sich aus der abstrakten algebraischen Beschreibung die gewohnte „klassische“ Raumzeit-Geometrie ergeben kann. Konkret sucht man nach einer Antwort, indem man eine größere Zahl an Variablen in die Korrespondenz zwischen E10 und der Stringtheorie einbaut. Hierbei gibt es zwei unterschiedliche Arten von Variablen. Zum einen sind dies weitere Veränderliche der Supergravitationstheorie, die im Wesentlichen mit einer größeren Variabilität der Raumzeit zusammenhängen. Zum anderen sind es neue Variablen aus der Stringtheorie, die alleine nicht in der reinen Supergravitationstheorie auftauchen. Wegen der exponentiell wachsenden Komplexität der E10-Algebra wird der Einbau dieser Variablen allerdings mit wachsender Anzahl immer schwieriger.

\section{DIE E10-STRUKTUR}

Die Mathematik behandelt E10 in der Theorie der hyperbolischen Kac-MoodyAlgebren. Viktor Kac und Robert Moody definierten sie 1968 unabhängig voneinander. Solche Algebren sind unendlichdimensionale Verallgemeinerungen endlichdimensionaler LieAlgebren. Letztere beschreiben die Symmetrien bestimmter einfacher endlichdimensionaler Räume (Kugelschalen). Eine ähnlich explizite Realisierung der Kac-Moody-Algebren ist nicht bekannt.

Unter den hyperbolischen KacMoody-Algebren nimmt die E10-
Struktur eine herausgehobene Stellung ein, da sie in gewissem Sinne die größte und symmetrischste ist. Auf die mögliche Relevanz der E10 in der theoretischen Physik hat erstmals 1980 der französische Theoretiker Bernard Julia hingewiesen. Der Name E10 stammt daher, dass E10 als Unterstruktur die sogenannte endlichdimensionale exzeptionelle Lie-Algebra E8 enthält, die eines der faszinierendsten Objekte der klassischen Lie-Theorie ist [7]. Die „10“ verweist auf das zehndimensionale Billard, das man mit der E10-Struktur assoziieren kann. 
Es gibt ermutigende Anzeichen dafür, dass es möglich sein könnte, sämtliche Variablen der Allgemeinen Relativitätstheorie in den E10-Rahmen einzubauen. Dies würde zu einer Wiedereinführung der gesamten Raumzeit über den unendlichdimensionalen E10-Raum führen. Damit bekäme man erstmals ein konkretes Beispiel einer Theorie, in dem die Raumzeit „emergent“ ist - also „von selbst“ aus einer Grundstruktur auftaucht, in der sie zunächst nicht manifest enthalten ist. Würde sich diese Hypothese bestätigen, dann würde dies eine radikal neue Sicht auf die Struktur der Raumzeit bei den kleinsten Abständen liefern [10].

In diesem Zusammenhang wird übrigens kontrovers diskutiert, ob die resultierende Theorie genau der Supergravitationstheorie entsprechen wird oder ob man etwa geringfügige Korrekturen zulassen muss. Solche Korrekturen werden auch von der Stringtheorie vorhergesagt. Erste Untersuchungen haben tatsächlich die Konsistenz dieser Korrekturen mit der E10-Struktur belegt. Ferner wird man auch mit wesentlichen Modifikationen der Quantentheorie selbst rechnen müssen, da quantenmechanische Phänomene wie Superposition und Verschränkung wesentlich auf eine umgebende Raumzeit Bezug nehmen.

\section{Zusammenfassung}

Symmetrien spielen in den grundlegenden Theorien der Physik eine zentrale Rolle. Sie könnten helfen, eine Quantengravitation zu entwickeln, die erfolgreich Allgemeine Relativitätstheorie und Quantentheorie zusammenbringt. Gibt es ein Symmetrieprinzip, das diese Zusammenführung bewirkt? Die einzigartige mathematische E10-Struktur eröffnet tatsächlich einen neuen Zugang zur Quantengravitation. Mit ihr ließe sich eine neue Beschreibung von urknallartigen Singularitäten und Schwarzen Löchern finden. Das vollständige Verständnis des E10-Modells mit seinen Auswirkungen auf die Struktur der Raumzeit könnte tiefe Einblicke in grundlegende Symmetrien und Prinzipien der Welt eröffnen. Noch allerdings steht die theoretische Physik hier am Anfang.

\section{Stichworte}

E10-Symmetrie, Quantengravitation, Symmetrien, Eichsymmetrien, Singularität, Schwarzes Loch, Urknall, Standardmodell, Stringtheorie, Supergravitation, Kosmologie.

\section{Literatur}

[1] S. Weinberg, Die ersten drei Minuten, Piper, München 1997.

[2] G. Hasinger, Das Schicksal des Universums: Eine Reise vom Anfang zum Ende, C.H. Beck, München 2007.

[3] Physik in unserer Zeit 2008, 39(3), 116.

[4] C. Lämmerzahl, Physik in unserer Zeit 2008, 39(3), 125.

[5] J. Louis, T. Mohaupt, S. Theisen, Springer Lecture Notes in Physics 2007, 721, 289.

[6] T. Damour, M. Henneaux, Phys. Rev. Lett 2001, 86, 4749.

[7] H. Nicolai, Nature 2007, 447, 41.

[8] A. Kleinschmidt, H. Nicolai, Int. J. Mod. Phys. D 2006, 15, 1619.

[9] A. Kleinschmidt, M. Koehn, H. Nicolai, Phys. Rev D 2009, 80 , 061701.

[10] T. Damour, H. Nicolai, Int. J. Mod. Phys. D 2008, 17, 525.

\section{Die Autoren}

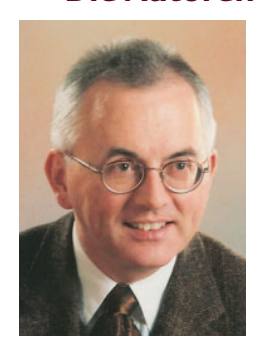

Hermann Nicolai ist Direktor am Max-Planck-Institut für Gravitationsphysik (Albert-Einstein-Institut) in Golm. Er forscht auf dem Gebiet der Quantengravitation und der Vereinigten Theorien. 2010 erhielt er die Einstein-Medaille der Berner Albert-EinsteinGesellschaft.

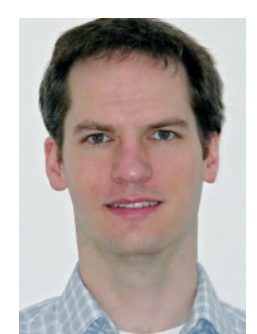

Axel Kleinschmidt forscht mit Unterstützung des belgischen Fonds de la Recherche Scientifique an der Université Libre de Bruxelles an Symmetrien in Gravitationstheorien und Quanteneffekten in der Stringtheorie.

\section{Anschriften}

Prof. Dr. Hermann Nicolai, Max-Planck-Institut für Gravitationsphysik, Am Mühlenberg 1, 14476 Potsdam. Hermann.Nicolai@aei.mpg.de

Dr. Axel Kleinschmidt, Université Libre de Bruxelles, Service de Physique Théorique et Mathématique CP231, Boulevard du Triomphe, 1050 Bruxelles, Belgien.Axel.Kleinschmidt@ulb.ac.be 